\title{
HD Chest
}

National Cancer Institute

\section{Source}

National Cancer Institute. HD Chest. NCI Thesaurus. Code C159518.

A proprietary combination of PET/CT hardware and software designed to provide

relatively motion-free images with higher count statistics for improved image quality and small lesion conspicuity. Using a highly automated, amplitude-based gating technology,

HD Chest adapts to every patients breathing and automatically selects the optimal data range with the least amount of motion and the highest count statistics, producing images the chest and upper abdomen in high-definition without respiratory motion. 